\title{
Separation and Identification of Glycan Anomers Using Ultrahigh-Resolution lon Mobility Spectrometry and Cryogenic lon Spectroscopy
}

\author{
Stephan Warnke, Ahmed Ben Faleh, Valeriu Scutelnic ${ }^{\dagger}$, and Thomas R. Rizzo* \\ Laboratoire de Chimie Physique Moléculaire, École Polytechnique Fédérale de Lausanne, EPFL SB ISIC \\ LCPM, Station 6, CH-1015 Lausanne, Switzerland
}

KEYWORDS: glycans, carbohydrates, anomers, glucose, ion mobility spectrometry, ion spectroscopy, mass spectrometry, SLIM

\begin{abstract}
The analysis of carbohydrates, or glycans, is challenging for established structure-sensitive gas-phase methods. The multitude of possible stereo-, regio- and structural isomers make them substantially more complex to analyze than DNA or proteins, and no one method is currently able to fully resolve them. While the combination of tandem mass spectrometry (MS) and ion mobility spectrometry (IMS) have made important inroads in glycan analysis, in many cases this approach is still not able to identify the precise isomeric form. To advance the techniques available for glycan analysis we employ two important innovations. First, we perform ultrahigh-resolution mobility separation using structures for lossless ion manipulations (SLIM) for isomer separation and pre-selection. We then complement this IMS-MS stage with a cryogenic IR spectroscopic dimension, since a glycan's vibrational spectrum provides a fingerprint that is extremely sensitive to the precise isomeric form. Using this unique approach in conjunction with oxygen18 isotopic labelling, we show on a range of disaccharides how the two $\alpha$ and $\beta$ anomers that every reducing glycan adopts in solution can be readily separated by mobility and identified based on their IR spectra. In addition to highlighting the power of our technique to detect minute differences in the structure of isomeric carbohydrates, these results provide the means to determine if and when anomericity is retained during collision-induced dissociation (CID) of larger glycans.
\end{abstract}




\section{INTRODUCTION}

The diversity in both the structure and function of glycans is perhaps unparalleled in biological systems. As individual molecules or as decoration on proteins and lipids, they play a vital role in a myriad of physiological processes and take active part in the molecular choreography that represents life [1-4]. The paramount importance of glycans is not, however, reflected in the current state of the analytical tools available for their analysis. Techniques combining liquid chromatography (LC) and mass spectrometry (MS) [5, 6], which allow for routine sequencing of proteins, have difficulties to provide rapid structural analysis of carbohydrates [7-9]. These difficulties arise from the many levels of isomerism that glycans possess [2]: (1) their monosaccharide constituents are often isomeric, differing only in the stereochemistry of a single carbon atom; (2) multiple $\mathrm{OH}$ groups of a single monosaccharide provide a variety of potential attachment points for the next subunit, resulting in isomers that differ in their linkage position as well as allowing the formation of branched structures; (3) the anomeric $\left(\mathrm{C}_{1}\right)$ carbon where the glycosidic linkage is realized represents another stereocenter, which results in each glycosidic bond being in either $\alpha$ or $\beta$ configuration. In addition, each glycan with a free reducing-end $\mathrm{OH}$ will be present in solution in both its $\alpha$ and $\beta$ anomeric form at ratios determined by the equilibrium of the mutarotation reaction that leads to their interconversion (Fig. 1a) [10]. It is this immense isomeric heterogeneity throughout the glycome that represents a major bottleneck for the development of robust, structure-sensitive, highthroughput analytical techniques and workflows.

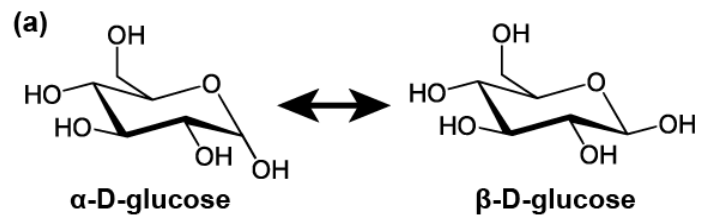

(b)

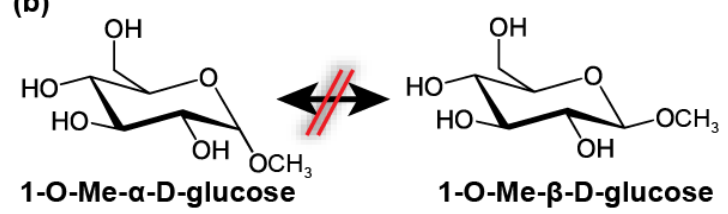

Figure 1: (a) Mutarotation of the $C_{1}$ carbon leads to an equilibrium between the $\alpha$ and $\beta$ anomers of reducing carbohydrates in solution. (b) The carbohydrates' reducing end is locked in $\alpha$ or $\beta$ configuration when the respective hydroxy group is methylated.

Ion mobility spectrometry $[11,12]$ (IMS), which is based on the difference in size and shape of a molecular ion when it is subjected to collisions with an inert buffer gas, is potentially sensitive to all kinds of different isomers. First studies using IMS-MS methods to separate glycan isomers were promising [13, 14], and these have been followed by more systematic investigations [15] of the feasibility of this approach for routine glycan analysis. This included the use of IMS-MS/MS methods [16, 17], where structural information can be obtained by fragment analysis of mobility-separated precursor ions in analogy to established LC-MS/MS workflows. Despite its promise, IMS alone cannot identify the subtlest forms of glycan isomerism, such as the difference in stereochemistry of a single carbon atom in an oligosaccharide.

Infrared (IR) spectroscopy of gas-phase molecular ions, on the other hand, can be exquisitely sensitive to the smallest of structural details $[18,19]$. Carbohydrates possess hydroxy groups, amines, and other substituents that can serve as structural probes because their frequencies will be highly characteristic of their local environment. A vibrational spectrum will therefore contain information about the structure-defining hydrogen-bonding network within each molecule. Aided by quantum mechanical calculations, IR spectroscopy becomes the ideal method for precise structural analysis of smaller sugar molecules [20-24], with the size-limiting factor being the computational methods available on the one hand and increasingly congested experimental spectra as the molecules become larger on the other. Nevertheless, a vibrational spectrum will always represent a characteristic fingerprint for a given carbohydrate structure. This realization initiated a series of studies that explored the sensitivity of different spectroscopic schemes to the various forms of carbohydrate isomerism [25-30], with the idea that an unknown carbohydrate compound could be identified by comparing its fingerprint spectrum to those from a database, bringing spectroscopy closer to becoming a routine-analysis tool.

Spectral congestion needs to be minimized for IR fingerprinting to be applicable as generically as possible. Considerably narrower bandwidths can be achieved when the number of populated vibrational states within the molecules are reduced by cooling them to low temperatures and applying, for example, messenger-tagging spectroscopic schemes [31-34], which yield linear absorption spectra. The applicability of cryogenic ion spectroscopy to carbohydrates has recently been demonstrated using both low-temperature ion traps [26, 27], and liquid helium droplets [25], to cool the ions. While decreasing the thermal internal energy distribution before spectral analysis is a prerequisite for resolved absorption bands, the isomeric heterogeneity of glycans will invariably result in the superposition of unique fingerprint spectra. In this case, the physical separation of isomers prior to spectroscopic investigation will greatly simply the 
measured IR spectra and their analysis. Liquid chromatography is one of the most commonly applied techniques for carbohydrate isomer separation, but a mismatch in experimental timescales renders a combination of LC with gas-phase spectroscopic methods a challenging task. Nevertheless, this approach has been recently employed in a study of isomeric disaccharides using the stop-flow technique to extend the elution time of an LC-separated compound to that needed to acquire a room-temperature IR multiple photon dissociation spectrum [35].

The timescale of IMS-MS, on the other hand, is ideally suited for combination with spectroscopy, and the potential of IMS to resolve small differences in size of isomeric molecules prior to their spectral analysis makes this approach particularly appealing [36, 37]. We have previously demonstrated how orthogonal MS, IMS, and IR spectroscopic data of carbohydrates could serve as reference for identification of glycans from unknown samples and even from mixtures $[27,38]$. We first used a drift-tube IMS instrument coupled to a cryogenic ion trap to measure the collision cross sections (CCS) of isomeric disaccharides prior to their spectroscopic analysis [26, 27], however it became evident that the IMS resolution provided by our $2 \mathrm{~m}$ drift tube would not be sufficient to resolve most of these species from a mixture. By variation of the charge state of the investigated glycans from protonated to deprotonated [39] or by using different metal ion adducts as charge carriers [40-42] it is possible to enhance IMS separation of specific glycan mixtures, however, a generally applicable method has not been established to date. Our approach to separate glycan isomers as completely as possible before spectroscopic interrogation employs a newly constructed instrument in which we combine ultrahighresolution IMS, using structures for lossless ion manipulation (SLIM) [43, 44], with a cryogenic ion trap and a time-offlight (TOF) MS to allow messenger-tagging IR spectroscopy of CCS- and m/z-selected glycans [38, 45]. Using SLIM travelling-wave ion-mobility, we have achieved a resolution of over 500 by cycling ions multiple times through a serpentine path, providing an extended drift length not feasible with conventional IMS methods [46].

In this paper we demonstrate the ability to resolve and identify $\alpha$ and $\beta$ anomers of reducing glycans using the combination of high-resolution SLIM IMS and cryogenic IR spectroscopy. We use selective oxygen-18 isotopic substitution to identify the anomeric $\mathrm{OH}$ band in the IR spectrum and assign each anomer by comparison with the spectra of the corresponding anomerically pure methylated species. While the identification of carbohydrate anomers can been achieved by other techniques, such as NMR [47], our ability to separate and identify such species in a mass spectrometer on a millisecond timescale is particularly significant in view of the recent evidence for retention of anomericity of a glycosidic bond upon collision-induced dissociation (CID) [28]. Application of an IMS-MS-MS-IR spectroscopic workflow to the $\mathrm{C}_{\mathrm{n}}$-fragments of glycans should be able to verify under which conditions such anomeric retention occurs. This could then be incorporated into a CID-based sequencing scheme to determine the stereochemistry of glycosidic linkages.

\section{MATERIALS AND METHODS}

Samples. Samples were purchased from Dextra (UK) or Carbosynth (UK) and used without further purification. Solvents were purchased from Sigma-Aldrich. For electrospray ionization, samples were dissolved in water/methanol $(\mathrm{v} / \mathrm{v} 50 / 50)$ to yield a final concentration of $50-100 \mu \mathrm{M}$. To enhance the abundance of the here-investigated sodiated species, we added 1.5 equivalents of $\mathrm{NaCl}$ to the solution. Oxygen-18 isotope labeling of disaccharides was achieved by dissolving samples in $\mathrm{H}_{2}{ }^{18} \mathrm{O}$ and incubating them at room temperature for three days. It is known that only the anomeric $\mathrm{OH}$ at the reducing end exchanges under these conditions [48].

Ion-mobility-selective IR spectroscopy. We use travelling-wave (TW) ion mobility implemented with structures for lossless ion manipulation (SLIM), developed by Smith and coworkers [43, 44], where the TW potentials created between a mirrored pair of printed circuit boards $(15 \times 15 \mathrm{~cm}$ in size) propel ions through a cyclic serpentine path $(1.8$ $\mathrm{m}$ single pass) to achieve high mobility resolution. The number of cycles $n$ can be varied using an electrostatic switch [46]. A schematic of our instrument is shown in Fig. 2a, and a detailed description can be found elsewhere [38].

Briefly, ions are electrosprayed using commercial nano-emitters (Thermo Fisher) and transferred into the instrument through a capillary. A dual-stage ion funnel assembly [49] collimates and stores ions in the hour-glass section of the second funnel before pulses of ions, $120 \mu$ s in duration, are released into the ion-mobility region where different species are separated based on their CCS. An example of the instrument's resolution is displayed in Fig $2 \mathrm{~b}$, where the separation and peak widths of the peptide ions GRGDS ${ }^{2+}$ and $\mathrm{SDGRG}^{2+}$ were investigated as a function of separation cycles $n$. The resulting curve follows the expected $n^{1 / 2}$ dependency [50] and reaches a resolution of approximately 500 at $n=28$ cycles.

(a)

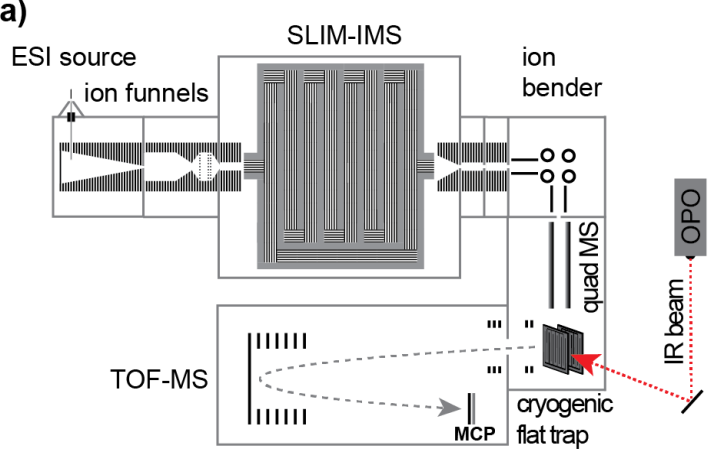

(b)

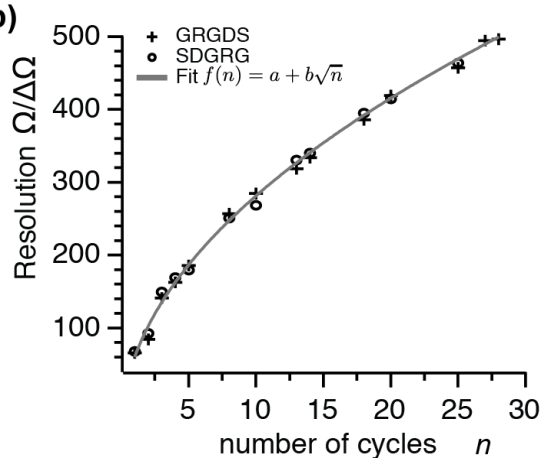


Figure 2: (a) Schematic of the IMS-MS-IR spectroscopy instrument that uses structures for lossless ion manipulation (SLIM) for ultrahigh resolution ion-mobility separation prior IR spectroscopic analysis in a planar, cryogenic trap. (b) The instrument's IMS resolution as a function of the number of separation cycles, $n$, determined for the peptides GRGDS ${ }^{2+}$ and $\mathrm{SDGRG}^{2+}$.

After separation by their drift time, ions are ejected from the SLIM board, pass through a mass-resolving quadrupole, and sent to the cryogenic ion trap, which is held at a temperature of $40 \mathrm{~K}$. Collisions with the cold buffer gas $\left(10 \% \mathrm{~N}_{2}\right.$ in $\mathrm{He}$ ) leads to cooling and formation of nitrogen-tagged ions, allowing the application of messenger-tagging spectroscopy [31-34]. A single laser pulse (10 mJ) from an infrared optical parametric oscillator (OPO) irradiates the trapped ions before they are pulsed into the TOF region for $\mathrm{m} / \mathrm{z}$ analysis. An IR spectrum is obtained by monitoring the wavelength-dependent dissociation yield of the nitrogen-tagged molecular ions. A single laser scan in the wavelength range reported here requires 6-8 minutes, and the spectra represent the average of two such scans.

\section{RESULTS AND DISCUSSION}

IMS of glycans. In analogy to bimodal distributions in LC chromatograms that are often reported for glycans, ion mobility arrival time distributions (ATDs) can also exhibit multiple features that were speculated to originate from the respective $\alpha$ and $\beta$ anomers [30, 45, 51, 52], however, identification of the two anomeric forms has remained elusive. In the present study we investigated a set of roughly 20 different sodiated glycans ranging from small mono- and disaccharides to larger oligosaccharides using our ultrahigh-resolution IMS instrument, a subset of which is displayed in Fig. 3a. Most interestingly, all investigated glycans with a free reducing-end $\mathrm{OH}$ exhibit two features in their ATD. For the three disaccharides displayed here, seven separation cycles were necessary to resolve these different species, whereas nine cycles were required to resolve two different features of glucose (top panel). Other disaccharides and larger oligosaccharides require different number of separation cycles in the SLIM section (i.e., different IMS resolution) to separate two features (see Fig. S1 in the Supporting Information SI).

(a) Reducing sugars

(b) Non-reducing sugars
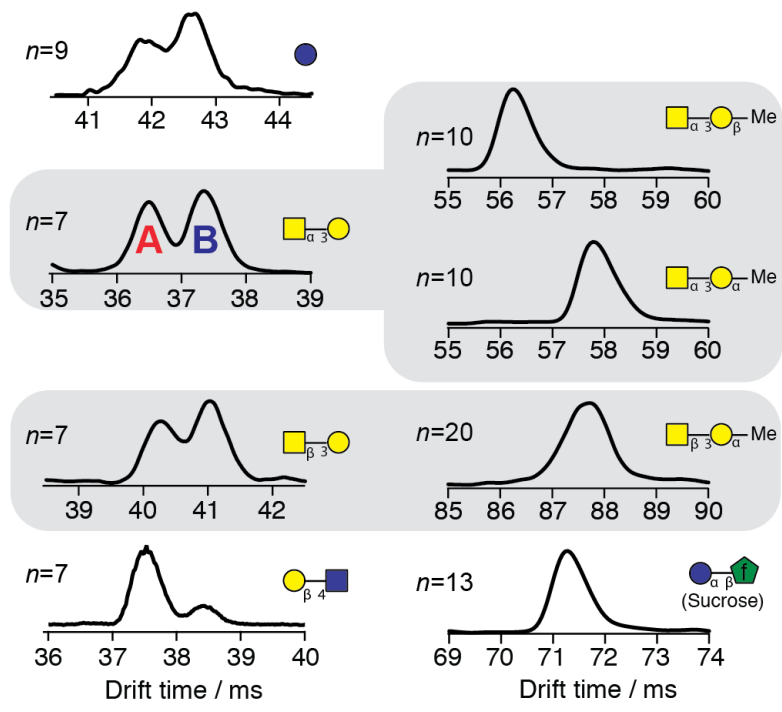

Figure 3: IMS arrival time distributions of reducing (a) and non-reducing glycans (b) at high IMS resolving power settings. The label $n$ indicates the number of IMS cycles employed for separation. The structure of glycans is shown using the CFG nomenclature.

Conversely, when the hydroxy group at the reducing end of a glycan is methylated, we observe only one peak in the ATDs, even at the higher resolving power reached after 20 separation cycles (Fig. 3b). The absence of a second species can be interpreted by the locking of the reducing-end $\mathrm{C}_{1}$ atom upon methylation, blocking the mutarotation process that is responsible for coexisting $\alpha$ and $\beta$ anomers in solution (Fig. 1b). Table sugar (sucrose) is also unable to form anomers, since the disaccharide is formed by $\mathrm{C}_{1}-\mathrm{C}_{1} \alpha-\beta$ glycosidic linkage of fucose and glucose and, consequently, has no free reducing-end hydroxy group. Its ATD in the bottom panel of Fig. $3 \mathrm{~b}$ shows only a single feature after 13 cycles, corresponding to an IMS resolution of approximately 300. In light of the observation that glycans with a free $\mathrm{OH}$ at the reducing end always seem to exhibit two peaks in their ATD, we used cryogenic ion spectroscopy to identify these species.

Spectroscopic identification of anomers. The two mobility-separated species of sodiated GalNAc- $\alpha-(1-3)-G a l$ (labeled $\mathbf{A}$ and $\mathbf{B}$ in the ATD in Fig. 3a) were selected by electrostatic deflection and loaded separately into the cryogenic trap where their infrared (IR) spectrum is measured. The results are displayed in Fig. $4 \mathrm{~b}$ and e for species $\mathbf{A}$ and $\mathbf{B}$, respectively. Signals in the wavenumber range below $3550 \mathrm{~cm}^{-1}$ can be attributed to strongly hydrogen-bonded $\mathrm{OH}$ and $\mathrm{NH}$ oscillators, while relatively free $\mathrm{OH}$ vibrations are expected to occur from $3600 \mathrm{~cm}^{-1}$ and above [20]. 


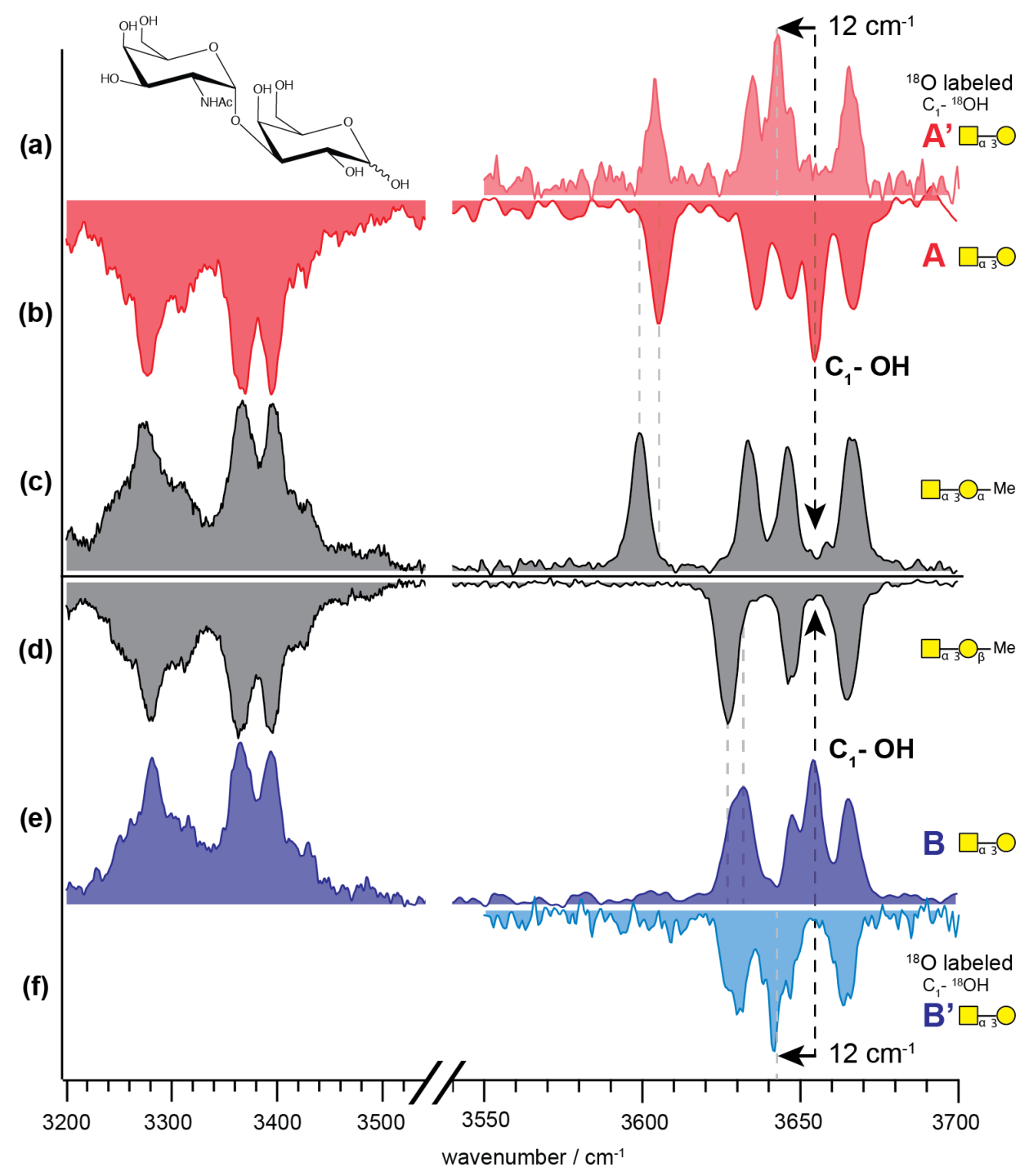

Figure 4: IR spectra of mobility-selected species A and B of sodiated GalNAc- $\alpha(1-3)-\mathrm{Gal}\left((\mathbf{b})\right.$ and (e)) and their $\mathrm{C}_{1}{ }^{-18} \mathrm{OH}$ labelled equivalents $\left((\mathbf{a})\right.$ and (f)) and the corresponding $\mathrm{C}_{1}$-O-methylated species with the $\mathrm{C}_{1}$ locked in $\alpha(\mathbf{c})$ or in $\beta$ (d) configuration. The $\mathrm{C}_{1}-\mathrm{OH}$ stretch vibration at $3655 \mathrm{~cm}^{-1}$ is clearly identified by the ${ }^{18} \mathrm{O}$-labeling experiment.

The high sensitivity of IR spectroscopy to smallest structural details are reflected in these spectra. While most absorption bands appear at the same wavenumber with almost identical relative intensities between the spectra of the two drift peaks, other signals, such as the band around $3600 \mathrm{~cm}^{-1}$ for species $\mathbf{A}$ and the broader absorption at $3630 \mathrm{~cm}^{-1}$ for species $\mathbf{B}$, are clearly characteristic of each structure. We expect a total of eight $\mathrm{OH}$ and $\mathrm{NH}$ oscillators for these molecules, which coincides with the number of resolved absorption bands in the spectrum of $\mathbf{A}$. In the spectrum of $\mathbf{B}$ there appear to be only 7 bands, but the broad absorption signal around $3630 \mathrm{~cm}^{-1}$ appears to be unresolved in this spectrum.

Proving the hypothesis that the two mobility-separated glycan species represent the $\alpha$ and $\beta$ anomers requires a deeper analysis of the IR spectra. The band corresponding to the anomeric $\mathrm{C}_{1}-\mathrm{OH}$ oscillator can be identified using oxygen-18 isotopic labelling, which should induce a spectral redshift. Indeed, a redshift of $12 \mathrm{~cm}^{-1}$ corresponding to the change in reduced mass of the respective $\mathrm{O}-\mathrm{H}$ oscillator can be observed for the absorption band at $3655 \mathrm{~cm}^{-1}$ as the only noticeable change when comparing spectra $\mathbf{A}$ and $\mathbf{B}$ with those of their $\mathrm{C}_{1}{ }^{-1}{ }^{18} \mathrm{O}$ labelled analogs $\mathbf{A}^{\prime}$ and $\mathbf{B}^{\prime}$ in Fig. $4 \mathrm{a}$ and $\mathrm{f}$, respectively. We thus identify the band at $3655 \mathrm{~cm}^{-1}$ in the spectrum of both mobility-separated species as that from the anomeric $\mathrm{OH}$.

Infrared spectra of the $\mathrm{C}_{1}$-methylated species of the same glycan with the $\mathrm{C}_{1}$-configuration locked in its $\alpha$ and $\beta$ position are shown in Fig. $4 \mathrm{c}$ and d, respectively. Most noticeably, the absorption band at $3655 \mathrm{~cm}^{-1}$ attributed to the $\mathrm{C}_{1}-\mathrm{OH}$ oscillator of the unsubstituted species is absent in these spectra, which is a direct result of the $\mathrm{C}_{1}-\mathrm{O}-\mathrm{CH}_{3}$ substitution. Moreover, the spectra of the methylated species resemble those of the corresponding unsubstituted species in the position and relative intensities of the remaining absorption bands. It is particularly striking that for each one of the two mobility-separated, unsubstituted species, its spectrum matches that of one of the methylated molecules to within a few $\mathrm{cm}^{-1}$. This leads to the conclusion that species $\mathbf{A}$ represents the $\alpha$ anomer and species $\mathbf{B}$ represents the $\beta$ anomer of GalNAc- $\alpha(1-3)$-Gal. The same strategy to identify mobility-separated glycan anomers was applied to two additional disaccharides with similar results (see Fig. S2 and S3 in the Supporting Information). 
Monitoring of glucose mutarotation. To further substantiate our ability to separate glycan anomers on a sub-second timescale, we have used it to monitor the mutarotation process in glucose. When a sample of freshly prepared, anomerically pure $\alpha$-D-glucose is injected into our instrument, we observe a single IMS feature for singly sodiated ions after nine separation cycles (Fig. 5a, bottom).

(a)

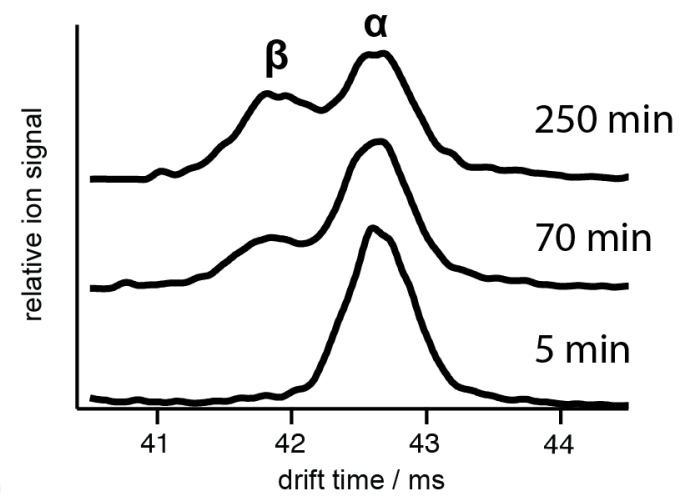

(b)

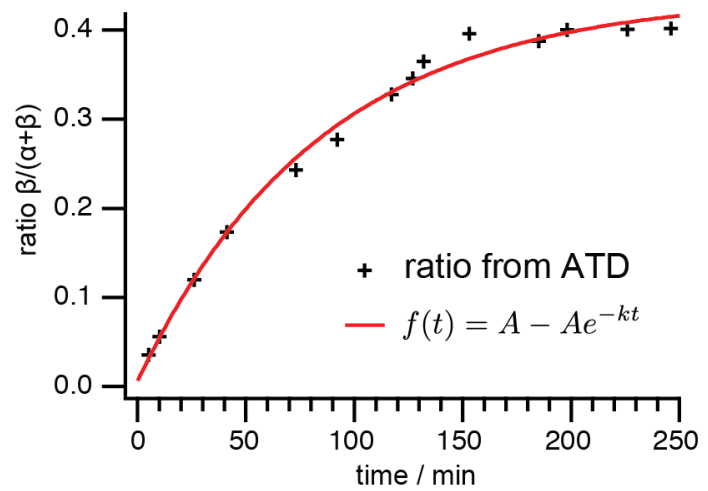

Figure 5: (a) ATDs of a sample of $\alpha$-D-Glucose at different times after sample preparation after $n=9$ separation cycles. (b) Relative ratios of the drift signals corresponding to the $\alpha$ and $\beta$ anomers as a function of time after sample preparation and the fit function $f(t)$ according to Eq. S4 in the supporting information.

After several minutes, a second feature at earlier drift times continues to increase in intensity until an equilibrium in relative signal intensities is reached after several hours (Fig. 5a, other traces). Given that $\alpha$-D-glucose will form its $\beta$ anomer in an equilibrium reaction on the observed timescales, it is likely that the two observed mobility-separated species represent these $\alpha$ and $\beta$ anomers, and an IR spectroscopic analysis of the two species separately and comparison of the resulting spectra to published reference spectra [20] confirms this assumption (Fig. S4, supporting information).

The IMS separation technique can thus be used to determine rate constants for the dynamic conversion reaction $\alpha$-D-glucose $\rightleftharpoons_{k_{\beta}}^{k_{\alpha}} \beta$-D-glucose by simply monitoring the formation of the $\beta$ species in real time. The ratio $\beta /(\beta+\alpha)$ of the relative intensities of the two signals in the ATD are plotted in Fig. $5 \mathrm{~b}$ as a function of time after sample preparation. The conversion can be treated as a first order reaction $[53,54]$ and the resulting differential rate equation describing the concentration of the $\beta$ species can be solved analytically by a function of the form $f(t)=A-A e^{-k t}$, with the observable reaction rate $k=k_{\alpha}+k_{\beta}$. This function is represented by the solid red curve in Fig. $5 \mathrm{~b}$ using $A$ and $k$ as fit parameters, which can then be used to obtain the rate constants $k_{\alpha}$ and $k_{\beta}$ (for details see supporting information) [53]. At the temperature and solvent conditions used here $\left(25^{\circ} \mathrm{C}, \mathrm{MeOH} / \mathrm{H}_{2} \mathrm{O} 50 / 50\right)$ we determined $k_{\alpha}=0.88 \times 10^{-4} \mathrm{~s}^{-1}$ and $k_{\beta}=1.13 \times 10^{-4} \mathrm{~s}^{-1}$, in agreement with previously published values $[53,55]$.

\section{CONCLUSIONS}

We demonstrate the power of a combined ultrahigh-resolution IMS and cryogenic IR spectroscopic approach to separate and identify glycan anomers of the reducing end. Cyclic IMS, which we realize using structures for lossless ion manipulation (SLIM), provides the necessary resolving power to distinguish two drift features of every mono- and disaccharide with a free reducing end investigated in our laboratory to date. The addition of an IR spectroscopic dimension allows us to probe the intramolecular hydrogen bonding network of the $\mathrm{m} / \mathrm{z}$ - and mobility-selected glycan species and is used here to identify the $\alpha$ and $\beta$ anomers. These results highlight the benefit of ultrahigh-resolution IMS as a tool for rapid separation of different glycan structures on the one hand, and illustrates the exquisite sensitivity of highresolution vibrational spectroscopy to smallest structural differences on the other. The ability to separate and identify glycan anomers in the gas phase is particularly significant for studying the retention of anomericity of a glycosidic bond upon collision-induced dissociation (CID) [28]. Application of an IMS-MS-MS-IR spectroscopic workflow to the $C_{n^{-}}$ fragments of glycans should be able to verify under what conditions such anomeric retention occurs. This could then be 
incorporated into a CID-based sequencing scheme to determine the stereochemistry of glycosidic linkages, which is particularly important in carbohydrate research.

\section{AUTHOR INFORMATION}

\section{Corresponding Author}

* Thomas R. Rizzo; e-mail: thomas.rizzo@epfl.ch

\section{Present Addresses}

$\uparrow$ Valeriu Scutelnic; Department of Chemistry, University of California, Berkeley, California 94720, USA

\section{ACKNOWLEDGMENT}

The authors thank the Swiss National Science Foundation (200020_165908), the European Research Council (788697GLYCANAL), and the EPFL for their generous support of this work. We would also like to acknowledge R.D. Smith and his group for helpful discussions on the implementation of the SLIM technology.

\section{REFERENCES}

1. Varki, A.; Biological roles of glycans. Glycobiology. 27, 3-49 (2017).

2. Varki, A., Cummings, R.D., Esko, J.D., Stanley, P., Hart, G.W., Aebi, M., Darvill, A.G., Kinoshita, T., Packer, N.H., Prestegard, J.H., Schnaar, R.L. and Seeberger, P.H., Essentials of Glycobiology (Cold Spring Harbor Laboratory Press, 2017), 3rd edn.

3. Varki, A.; Biological roles of oligosaccharides: all of the theories are correct. Glycobiology. 3, 97-130 (1993).

4. Dwek, R.A.; Glycobiology: Toward Understanding the Function of Sugars. Chemical Reviews. 96, 683-720 (1996).

5. Aebersold, R. and Mann, M.; Mass spectrometry-based proteomics. Nature. 422, 198-207 (2003).

6. Shi, Y., Xiang, R., Horváth, C. and Wilkins, J.A.; The role of liquid chromatography in proteomics. Journal of Chromatography A. 1053, 27-36 (2004).

7. Zaia, J.; Mass spectrometry of oligosaccharides. Mass Spectrom Rev. 23, 161-227 (2004).

8. Veillon, L., Huang, Y., Peng, W., Dong, X., Cho, B.G. and Mechref, Y.; Characterization of isomeric glycan structures by LC-MS/MS. Electrophoresis. 38, 2100-2114 (2017).

9. Marino, K., Bones, J., Kattla, J.J. and Rudd, P.M.; A systematic approach to protein glycosylation analysis: a path through the maze. Nat Chem Biol. 6, 713-723 (2010).

10. Pigman, W. and Isbell, H.S.; Mutarotation of Sugars in Solution*: Part I: History, Basic Kinetics, and Composition of Sugar Solutions. Advances in Carbohydrate Chemistry. 23, 11-57 (1968).

11. Bowers, M.T.; Ion mobility spectrometry: A personal view of its development at UCSB. Int J Mass Spectrom. 370, 75-95 (2014).

12. Lanucara, F., Holman, S.W., Gray, C.J. and Eyers, C.E.; The power of ion mobility-mass spectrometry for structural characterization and the study of conformational dynamics. Nat Chem. 6, 281-294 (2014).

13. Liu, Y. and Clemmer, D.E.; Characterizing oligosaccharides using injected-ion mobility/mass spectrometry. Anal Chem. 69, 2504-2509 (1997).

14. Lee, S., Wyttenbach, T. and Bowers, M.T.; Gas phase structures of sodiated oligosaccharides by ion mobility/ion chromatography methods. International Journal of Mass Spectrometry and Ion Processes. 167-168, 605-614 (1997).

15. Hofmann, J. and Pagel, K.; Glycan Analysis by Ion Mobility-Mass Spectrometry. Angewandte Chemie International Edition. 56, 83428349 (2017).

16. Hofmann, J., Stuckmann, A., Crispin, M., Harvey, D.J., Pagel, K. and Struwe, W.B.; Identification of Lewis and Blood Group Carbohydrate Epitopes by Ion Mobility-Tandem-Mass Spectrometry Fingerprinting. Anal Chem. 89, 2318-2325 (2017).

17. Li, H., Bendiak, B., Siems, W.F., Gang, D.R. and Hill, H.H., Jr.; Carbohydrate structure characterization by tandem ion mobility mass spectrometry (IMMS)2. Anal Chem. 85, 2760-2769 (2013).

18. Manz, C. and Pagel, K.; Glycan analysis by ion mobility-mass spectrometry and gas-phase spectroscopy. Curr Opin Chem Biol. 42, 1624 (2018).

19. Boyarkin, O.V.; Cold ion spectroscopy for structural identifications of biomolecules. International Reviews in Physical Chemistry. 37, 559-606 (2018).

20. Voss, J.M., Kregel, S.J., Fischer, K.C. and Garand, E.; IR-IR Conformation Specific Spectroscopy of Na(+)(Glucose) Adducts. J Am Soc Mass Spectrom. 29, 42-50 (2018).

21. Scutelnic, V. and Rizzo, T.R.; Cryogenic Ion Spectroscopy for Identification of Monosaccharide Anomers. J Phys Chem A. 123, 28152819 (2019).

22. Barnes, L., Schindler, B., Chambert, S., Allouche, A.-R. and Compagnon, I.; Conformational preferences of protonated N-acetylated hexosamines probed by InfraRed Multiple Photon Dissociation (IRMPD) spectroscopy and ab initio calculations. International Journal of Mass Spectrometry. 421, 116-123 (2017).

23. Contreras, C.S., Polfer, N.C., Oomens, J., Steill, J.D., Bendiak, B. and Eyler, J.R.; On the path to glycan conformer identification: Gasphase study of the anomers of methyl glycosides of N-acetyl-d-glucosamine and N-acetyl-d-galactosamine. International Journal of Mass Spectrometry. 330-332, 285-294 (2012).

24. Tan, Y., Zhao, N., Liu, J., Li, P., Stedwell, C.N., Yu, L. and Polfer, N.C.; Vibrational Signatures of Isomeric Lithiated N-acetyl-Dhexosamines by Gas-Phase Infrared Multiple-Photon Dissociation (IRMPD) Spectroscopy. J Am Soc Mass Spectrom. 28, 539-550 (2017).

25. Mucha, E., Gonzalez Florez, A.I., Marianski, M., Thomas, D.A., Hoffmann, W., Struwe, W.B., Hahm, H.S., Gewinner, S., Schollkopf, W., Seeberger, P.H., von Helden, G. and Pagel, K.; Glycan Fingerprinting via Cold-Ion Infrared Spectroscopy. Angewandte Chemie International Edition. 56, 11248-11251 (2017).

26. Khanal, N., Masellis, C., Kamrath, M.Z., Clemmer, D.E. and Rizzo, T.R.; Cryogenic IR spectroscopy combined with ion mobility spectrometry for the analysis of human milk oligosaccharides. Analyst. 143, 1846-1852 (2018).

27. Masellis, C., Khanal, N., Kamrath, M.Z., Clemmer, D.E. and Rizzo, T.R.; Cryogenic Vibrational Spectroscopy Provides Unique Fingerprints for Glycan Identification. Journal of the American Society for Mass Spectrometry. 28, 2217-2222 (2017).

28. Schindler, B., Barnes, L., Renois, G., Gray, C., Chambert, S., Fort, S., Flitsch, S., Loison, C., Allouche, A.R. and Compagnon, I.; Anomeric memory of the glycosidic bond upon fragmentation and its consequences for carbohydrate sequencing. Nature Communications. 8, 973 (2017).

29. Saparbaev, E., Kopysov, V., Yamaletdinov, R., Pereverzev, A. and Boyarkin, O.V.; Interplay of H-bonds with Aromatics in Isolated Complexes Identifies Isomeric Carbohydrates. Angewandte Chemie International Edition. doi:10.1002/ange.201902377 (2019). 
30. Hernandez, O., Isenberg, S., Steinmetz, V., Glish, G.L. and Maitre, P.; Probing Mobility-Selected Saccharide Isomers: Selective IonMolecule Reactions and Wavelength-Specific IR Activation. J Phys Chem A. 119, 6057-6064 (2015).

31. Wolk, A.B., Leavitt, C.M., Garand, E. and Johnson, M.A.; Cryogenic ion chemistry and spectroscopy. Acc Chem Res. 47, 202-210 (2014).

32. Leavitt, C.M., Wolk, A.B., Fournier, J.A., Kamrath, M.Z., Garand, E., Van Stipdonk, M.J. and Johnson, M.A.; Isomer-Specific IR-IR Double Resonance Spectroscopy of D2-Tagged Protonated Dipeptides Prepared in a Cryogenic Ion Trap. J Phys Chem Lett. 3, 10991105 (2012).

33. Masson, A., Kamrath, M.Z., Perez, M.A., Glover, M.S., Rothlisberger, U., Clemmer, D.E. and Rizzo, T.R.; Infrared Spectroscopy of Mobility-Selected H+-Gly-Pro-Gly-Gly (GPGG). Journal of the American Society for Mass Spectrometry. 26, 1444-1454 (2015).

34. Goebbert, D.J., Wende, T., Bergmann, R., Meijer, G. and Asmis, K.R.; Messenger-tagging electrosprayed ions: vibrational spectroscopy of suberate dianions. J Phys Chem A. 113, 5874-5880 (2009).

35. Schindler, B., Laloy-Borgna, G., Barnes, L., Allouche, A.R., Bouju, E., Dugas, V., Demesmay, C. and Compagnon, I.; Online Separation and Identification of Isomers Using Infrared Multiple Photon Dissociation Ion Spectroscopy Coupled to Liquid Chromatography: Application to the Analysis of Disaccharides Regio-Isomers and Monosaccharide Anomers. Anal Chem. 90, 11741-11745 (2018).

36. Warnke, S., Seo, J., Boschmans, J., Sobott, F., Scrivens, J.H., Bleiholder, C., Bowers, M.T., Gewinner, S., Schollkopf, W., Pagel, K. and von Helden, G.; Protomers of benzocaine: solvent and permittivity dependence. J Am Chem Soc. 137, 4236-4242 (2015).

37. Kamrath, M.Z. and Rizzo, T.R.; Combining Ion Mobility and Cryogenic Spectroscopy for Structural and Analytical Studies of Biomolecular Ions. Acc Chem Res. 51, 1487-1495 (2018).

38. Ben Faleh, A., Warnke, S. and Rizzo, T.R.; Combining Ultrahigh-Resolution Ion-Mobility Spectrometry with Cryogenic Infrared Spectroscopy for the Analysis of Glycan Mixtures. Anal Chem. 91, 4876-4882 (2019).

39. Struwe, W.B., Baldauf, C., Hofmann, J., Rudd, P.M. and Pagel, K.; Ion mobility separation of deprotonated oligosaccharide isomers evidence for gas-phase charge migration. Chemical Communications. 52, 12353-12356 (2016).

40. Fenn, L.S. and McLean, J.A.; Structural resolution of carbohydrate positional and structural isomers based on gas-phase ion mobilitymass spectrometry. Physical Chemistry Chemical Physics. 13, 2196-2205 (2011).

41. Huang, Y. and Dodds, E.D.; Ion mobility studies of carbohydrates as group I adducts: isomer specific collisional cross section dependence on metal ion radius. Analytical Chemistry. 85, 9728-9735 (2013).

42. Morrison, K.A., Bendiak, B.K. and Clowers, B.H.; Enhanced Mixture Separations of Metal Adducted Tetrasaccharides Using Frequency Encoded Ion Mobility Separations and Tandem Mass Spectrometry. Journal of the American Society for Mass Spectrometry. 28, 664677 (2017).

43. Hamid, A.M., Ibrahim, Y.M., Garimella, S.V., Webb, I.K., Deng, L., Chen, T.C., Anderson, G.A., Prost, S.A., Norheim, R.V., Tolmachev, A.V. and Smith, R.D.; Characterization of Traveling Wave Ion Mobility Separations in Structures for Lossless Ion Manipulations. Anal Chem. 87, 11301-11308 (2015).

44. Deng, L., Ibrahim, Y.M., Hamid, A.M., Garimella, S.V., Webb, I.K., Zheng, X., Prost, S.A., Sandoval, J.A., Norheim, R.V., Anderson, G.A., Tolmachev, A.V., Baker, E.S. and Smith, R.D.; Ultra-High Resolution Ion Mobility Separations Utilizing Traveling Waves in a 13 $\mathrm{m}$ Serpentine Path Length Structures for Lossless Ion Manipulations Module. Anal Chem. 88, 8957-8964 (2016).

45. Warnke, S., Ben Faleh, A., Pellegrinelli, R.P., Yalovenko, N. and Rizzo, T.R.; Combining ultra-high resolution ion mobility spectrometry with cryogenic IR spectroscopy for the study of biomolecular ions. Faraday Discussions. doi:10.1039/c1038fd00180d (2019).

46. Deng, L., Webb, I.K., Garimella, S.V.B., Hamid, A.M., Zheng, X., Norheim, R.V., Prost, S.A., Anderson, G.A., Sandoval, J.A., Baker, E.S., Ibrahim, Y.M. and Smith, R.D.; Serpentine Ultralong Path with Extended Routing (SUPER) High Resolution Traveling Wave Ion Mobility-MS using Structures for Lossless Ion Manipulations. Anal Chem. 89, 4628-4634 (2017).

47. Ryu, K.S., Kim, C., Park, C. and Choi, B.S.; NMR analysis of enzyme-catalyzed and free-equilibrium mutarotation kinetics of monosaccharides. Journal of the American Chemical Society. 126, 9180-9181 (2004).

48. Risley, J.M. and Van Etten, R.L.; Kinetics of oxygen exchange at the anomeric carbon atom of D-glucose and D-erythrose using the oxygen-18 isotope effect in carbon-13 nuclear magnetic resonance spectroscopy. Biochemistry. 21, 6360-6365 (2002).

49. Chen, T.C., Fillmore, T.L., Prost, S.A., Moore, R.J., Ibrahim, Y.M. and Smith, R.D.; Orthogonal Injection Ion Funnel Interface Providing Enhanced Performance for Selected Reaction Monitoring-Triple Quadrupole Mass Spectrometry. Analytical Chemistry. 87, 7326-7331 (2015).

50. Shvartsburg, A.A. and Smith, R.D.; Fundamentals of traveling wave ion mobility spectrometry. Anal Chem. 80, 9689-9699 (2008).

51. Nagy, G., Attah, I.K., Garimella, S.V.B., Tang, K., Ibrahim, Y.M., Baker, E.S. and Smith, R.D.; Unraveling the isomeric heterogeneity of glycans: ion mobility separations in structures for lossless ion manipulations. Chemical Communications. 54, 11701-11704 (2018).

52. Ujma, J., Ropartz, D., Giles, K., Richardson, K., Langridge, D., Wildgoose, J., Green, M. and Pringle, S.; Cyclic Ion Mobility Mass Spectrometry Distinguishes Anomers and Open-Ring Forms of Pentasaccharides. Journal of the American Society for Mass Spectrometry. (2019).

53. Lin, C.E., Yu, C.J., Chen, C.L., Chou, L.D. and Chou, C.; Kinetics of glucose mutarotation assessed by an equal-amplitude paired polarized heterodyne polarimeter. J Phys Chem A. 114, 1665-1669 (2010).

54. Ballash, N.M. and Robertson, E.B.; The Mutarotation of Glucose in Dimethylsulfoxide and Water Mixtures. Can. J. Chem. 51, 556-564 (1972).

55. Kendrew, J.C. and Moelwyn-Hughes, E.A.; The Kinetics of Mutarotation in Solution. Proceedings of the Royal Society of London. Series A, Mathematical and Physical Sciences. 176, 352-367 (1940).

56. Shen, Y.H., Tsai, S.T., Liew, C.Y. and Ni, C.K.; Mass spectrometry-based identification of carbohydrate anomeric configuration to determine the mechanism of glycoside hydrolases. Carbohydr Res. 476, 53-59 (2019). 